\title{
Profile of trebananib (AMG386) and its potential in the treatment of ovarian cancer
}

This article was published in the following Dove Press journal:

OncoTargets and Therapy

4 October 2014

Number of times this article has been viewed

\section{Michalis Liontos \\ Maria Lykka \\ Meletios-Athanasios \\ Dimopoulos \\ Aristotle Bamias}

Oncology Department, Therapeutics Clinic, Medical School, University of Athens, Athens, Greece
Correspondence: Aristotle Bamias Alexandra Hospital, 80 Vas Sofias Ave, Athens II528, Greece

Tel +30210338 I546

Fax +302132162511

Email abamias@med.uoa.gr
Abstract: Angiogenesis has been implicated in ovarian cancer pathogenesis. Bevacizumab, an anti-vascular endothelial growth factor monoclonal antibody, has recently been incorporated in ovarian cancer treatment in combination with chemotherapy both in a frontline setting and in disease recurrence. However, resistance eventually develops and treatment with bevacizumab is associated with increased risk for toxicities such as thromboembolic and hemorrhagic events, gastrointestinal perforation, and impaired wound healing, suggesting the need for new therapeutic approaches. Targeting of the angiopoietins/Tie2 pathway has gained accumulating interest during the last few years as a strategy to overcome bevacizumab resistance and toxicities. Trebananib is a first-in-class peptibody that inhibits angiopoietin 1 and 2 interaction with their receptor Tie2. The molecular profile of this agent, the preclinical data, and clinical studies demonstrating its efficacy in ovarian cancer are discussed in this review.

Keywords: trebananib, ovarian cancer, angiopoietins

\section{Introduction}

Ovarian cancer is the most lethal gynecological malignancy. Despite the fact that $70 \%$ of the women diagnosed with the disease will respond to frontline taxane/platinum compound combination chemotherapy, ultimately most patients will succumb to their disease. This underlines the medical need for the development of improved therapeutic solutions.

Recently, angiogenesis has been recognized as an important mechanism promoting ovarian cancer progression ${ }^{1,2}$ and intraperitoneal spread. ${ }^{3,4}$ On that basis, bevacizumab, a humanized anti-vascular endothelial growth factor (VEGF) monoclonal antibody, proved its clinical efficacy in ovarian $\operatorname{cancer}^{5,6}$ and has now gained regulatory approval for its use in combination with chemotherapy in both the frontline setting ${ }^{7,8}$ and platinum-sensitive ${ }^{9}$ recurrence of the disease. In addition, recent data showed significant efficacy also in platinum-resistant disease. ${ }^{10,11}$ Analysis of both Gynecologic Oncology Group 218 and International Collaboration on Ovarian Neoplasms 7 studies that used bevacizumab in combination with chemotherapy in the frontline setting indicates that addition of bevacizumab significantly prolonged progression-free survival (PFS), but, eventually, most patients recurred, thus still requiring effective treatments at relapse. Also, bevacizumab use is related to higher incidence of serious adverse events such as thromboembolic episodes, gastrointestinal perforations, hypertension, and impaired wound healing. ${ }^{12,13}$

These data denote the need to improve our understanding of the mechanisms of angiogenesis and to develop more efficient and less toxic treatment. Angiogenesis is one 
of the hallmarks of cancer. ${ }^{14,15}$ Formation of new vessels is a prerequisite for growing tumors to maintain supply of oxygen and nutrients. ${ }^{16}$ In solid tumors, six different mechanisms of new vessel formation have been recognized, namely sprouting angiogenesis, intussusceptive angiogenesis, the recruitment of endothelial progenitor cells, vessel cooption, vasculogenic mimicry, and lymphangiogenesis. ${ }^{17}$ The VEGF pathway is mainly implicated in sprouting angiogenesis. Anti-VEGF treatment blocks this mechanism, but concomitantly enhances alternative pathways of new vessel formation as is vessel cooption, that is driven by the angiopoietin (Ang)/Tie2 pathway (Figure 1). ${ }^{18,19}$ The latter signifies that novel agents targeting the Ang/Tie pathway could provide clinical benefit. Trebananib is the most clinically advanced inhibitor of this pathway, and its formulation, as well as available preclinical and clinical data focusing mainly on clinical cancer, are herein analyzed.

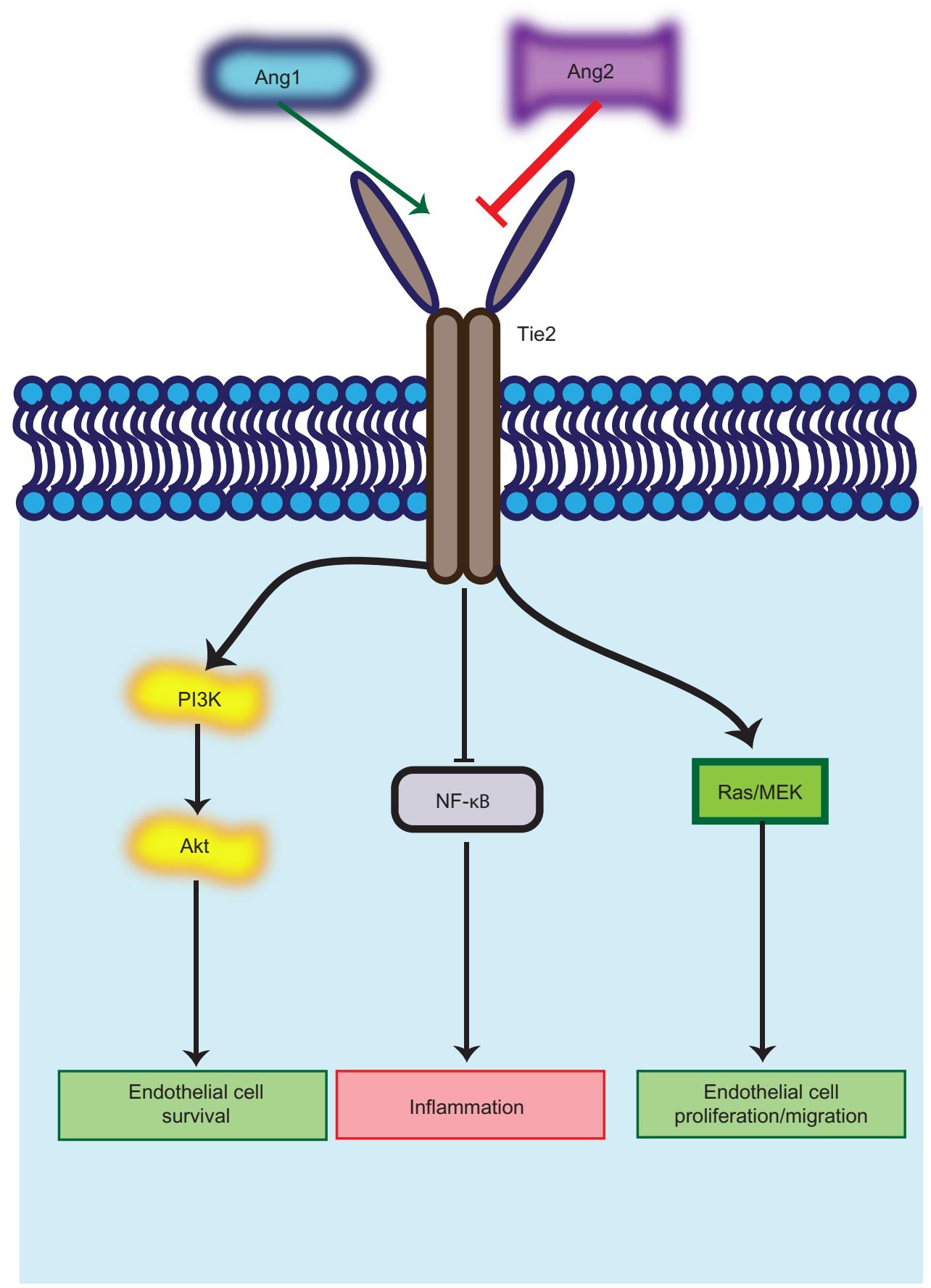

Figure I Angiopoietin (Ang)/Tie2 pathway and its role in vascular remodeling. Ang I binding to the Tie2 receptor activates prosurvival pathways, decreases endothelial cell permeability, and stabilizes vessels by recruiting pericytes. Ang2 acts antagonistically to the Angl/Tie2 binding, promoting sprouting angiogenesis through facilitating vascular endothelial growth factor-dependent proangiogenic pathways. 


\section{Trebananib profile}

Trebananib is a peptide inhibitor that neutralizes both Ang1 and Ang2 interaction with Tie2 receptor, reducing tumor angiogenesis. ${ }^{20}$ It belongs to a new class of therapeutic molecules called peptibodies, consisting of a biologically active peptide that is grafted to the $\mathrm{Fc}$ region of an immunoglobulin $\mathrm{G}$ (IgG). The active peptide of trebananib was initially discovered through a phage display library screening for Ang2 inhibitors. ${ }^{20}$ Active peptides selected through this process were subsequently fused to the $\mathrm{Fc}$ region of $\mathrm{IgG}$, allowing for increased stability and pharmacodynamic effect, ${ }^{21}$ and the generated molecules were again screened by enzymelinked immunosorbent assay for their ability to neutralize the Ang2-Tie2 interaction. Following this methodology, trebananib was developed (then named $2 \mathrm{xCon} 4[\mathrm{C}]$ ), demonstrating inhibitory activity for both $\mathrm{Ang} 2\left(\mathrm{IC}_{50} 0.023 \mathrm{nM}\right)$ and Ang1 $\left(\mathrm{IC}_{50} 0.9 \mathrm{nM}\right)^{20}$ interaction with Tie2.

\section{Trebananib in preclinical models}

Trebananib development is a successful example of the pathway from rationalized basic science to clinical therapeutics. Human Ang2 was initially cloned in 1997 and considered a naturally occurring antagonist of Ang1 and Tie 2. ${ }^{22}$ However, the concomitant analysis of Ang2 expression in adult human tissues revealed that Ang2 was present only in the ovary, placenta, and uterus, namely the predominant sites of vascular remodeling in adults. ${ }^{22}$ Most significantly, the pattern of Ang2 expression in conjunction with that of VEGF at the sites of vessel invasion indicated that Ang2 may allow endothelial cells to remain more responsive to proangiogenic VEGF signaling. ${ }^{22}$ This was confirmed in preclinical models from xenografts of human colon and gastric cancer cell lines overexpressing Ang2. ${ }^{23,24}$ Therefore, an attractive therapeutic target was recognized to disrupt new vessel sprouting and concomitantly proangiogenic signaling of VEGF. Since Tie2 inhibition affected both Ang1 and Ang2 signaling, selective Ang2-Tie2 interaction disruption was targeted, leading to trebananib development.

Evaluation of trebananib growth inhibitory activity was initially conducted in xenograft models from the human epidermoid cancer cell line A431 and the human colon cancer cell line Colo205. A431 cells overexpress epidermal growth factor receptor and also display vessel selective Ang2 expression. In both models, trebananib use resulted in tumor regression that was mechanistically linked to Ang2-mediated suppression of endothelial cell proliferation and increased apoptosis of tumor cells. ${ }^{20}$ The effect of trebananib was present both when the agent was infused before establishment of clinically detected xenograft tumors ( 3 days post cell injection) and when the drug infusion was started after establishment of evident tumors. Finally, the antiangiogenic effect of trebananib was confirmed in a VEGF-induced corneal vascularization rat model.

Although trebananib was developed as a selective antiAng2 agent, later experiments revealed that its clinical efficacy was attributed to both its anti-Ang1 and Ang2 activity. Trebananib exerted a greater antiproliferative effect in comparison with selective Ang1 (mL4-3) and Ang2 (L1-7[N]) inhibitors in colon cancer cell line xenograft models, suggesting that the Ang2 antagonism may unmask the antineoplastic effect of Ang1 inhibition. ${ }^{25}$ Histologically, selective Ang2 inhibition in the Colo205 xenograft model reduces the total number of vessels in the tumor, but the remaining vessels are normalized due to the unopposed Ang1 activity. ${ }^{26-28}$ The remaining stabilized vessels provide the tumor with adequate nutrients and oxygen, while normalization prevents further vessel regression decreasing the anticancer potential of selective Ang2 inhibitors. ${ }^{29,30}$ Therefore, concomitant Ang1 and Ang2 inhibition by trebananib potentiates the agent's antitumor effect not only by reducing the number of vessels in the tumor but also by leading to the regression of the remaining ones. ${ }^{26}$ Interestingly, the extent of tumor regression achieved with trebananib was comparable with the established antiangiogenic agent bevacizumab, while combination of these two agents exerted a more pronounced effect. ${ }^{25}$ The latter provides evidence that the blockade of multiple pathways implicated in angiogenesis may result in a more favorable clinical outcome.

A final conclusion drawn by the preclinical evaluation of trebananib concerns its role in ovarian angiogenesis and possible implications in ovarian cancer. The ovary is one of the adult tissues subjected to continuous remodeling during ovulation and undergoes normal angiogenesis. Insights into the mechanisms of normal ovarian angiogenesis and differences with pathological angiogenesis noted in ovarian carcinomas could facilitate the rational design of new therapeutic approaches. As previously described, Ang2 expression has been detected in normal ovary. ${ }^{22}$ Analogously, Ang1 and Ang receptor Tie2 expression has also been shown in ovarian tissues. ${ }^{31}$ Early results indicated that Ang1 and Ang2 act antagonistically, but this relation is reversed by Ang2 inhibition and Ang1 leads to vessel normalization. However, experiments with specific Ang1 and Ang2 inhibitors in a hormone-induced model of ovarian follicular angiogenesis in mice indicated that inhibition of either Ang is sufficient to decrease ovarian angiogenesis and induce ovarian atrophy, suggesting that both these molecules play a synergistic 
proangiogenic role in ovary. The latter, as well as data showing substantial expression of the members of Ang/Tie2 pathway in ovarian carcinomas, ${ }^{31}$ further enhances the rationale for using trebananib in ovarian cancer treatment.

\section{Trebananin in Phase I/II clinical trials in ovarian cancer} Trebananib monotherapy Phase I studies

The encouraging preclinical data for trebananib previously described led to its testing in clinical trials. The first human study of trebananib was reported in a 2007 American Society of Clinical Oncology meeting, ${ }^{32}$ and the final results were later published in 2009. ${ }^{33}$ It was a Phase I open-label study with a dose escalating and a dose expansion phase aiming primarily at determining the safety and pharmacokinetics of trebananib as well as possible antitumor activity (all Phase I and II studies of trebananib enrolling ovarian cancer patients are summarized in Table 1).

In this trial, trebananib was administered as a 60 minute intravenous infusion (later decreased to 30 minutes) weekly. Thirty-two patients with advanced solid tumors were finally recruited in the study. According to study design, at the dose escalation phase, four patients were included sequentially in each of five dose cohorts $(0 \mathrm{mg} / \mathrm{kg}, 3 \mathrm{mg} / \mathrm{kg}, 1 \mathrm{mg} / \mathrm{kg}$, $3 \mathrm{mg} / \mathrm{kg}, 10 \mathrm{mg} / \mathrm{kg}$, and $30 \mathrm{mg} / \mathrm{kg}$ ) and were monitored for a period of 4 weeks for possible dose-limiting toxicities (DLTs).
After 4 weeks of treatment, tumor response was assessed, and if no disease progression was noted, trebananib administration was resumed at week 6 . At the expansion phase, ten patients were enrolled and received trebananib at $30 \mathrm{mg} / \mathrm{kg}$ weekly until disease progression. Patients recruited at lower doses were offered the $30 \mathrm{mg} / \mathrm{kg}$ dose at the expansion phase. No DLTs were reported for doses up to $10 \mathrm{mg} / \mathrm{kg}$, while one patient receiving trebananib at $30 \mathrm{mg} / \mathrm{kg}$ during the expansion phase died from respiratory arrest. Treatment-related toxicities were reported in 16 patients (50\%) and, apart from the death, were Grade 1 and 2. Most common adverse events were fatigue ( $25 \%$ of patients), peripheral edema ( $13 \%$ ), and nausea $(6 \%)$. Adverse events common in the treatment with anti-VEGF therapies were not of clinical significance in this study. No thromboembolic or bleeding events were reported, while Grade 1 and 2 hypertension was noted in two patients but was not attributed to trebananib treatment. Proteinuria was detected in eleven patients (six with Grade 2 and five with Grade 3) but was transient and resolved within 2 weeks.

In terms of efficacy, among the 29 patients with evaluable disease, one had a partial response (PR) and 16 had stable disease (SD) (four of them for $\geq 16$ weeks). Patients received a median of six weekly trebananib infusions. Notably, the patient who experienced PR with trebananib was suffering from refractory ovarian cancer and she withdrew from the study after 156 weeks remaining in PR. This patient also demonstrated the greatest tumor reduction with trebananib among

Table I Phase I/II studies with trebananib including ovarian carcinoma patients

\begin{tabular}{|c|c|c|c|}
\hline Drug & Regimen & Phase & Reference \\
\hline \multirow[t]{6}{*}{ Trebananib } & Cohort A: $0.3 \mathrm{mg} / \mathrm{kg}$ & 1 & Herbst et $\mathrm{al}^{33}$ \\
\hline & Cohort B: I mg/kg & & \\
\hline & Cohort C: 3 mg/kg & & \\
\hline & Cohort D: $10 \mathrm{mg} / \mathrm{kg}$ & & \\
\hline & Cohort E: $30 \mathrm{mg} / \mathrm{kg}$ & & \\
\hline & Expansion phase: $30 \mathrm{mg} / \mathrm{kg}$ & & \\
\hline \multirow[t]{3}{*}{ Trebananib } & Cohort A: $3 \mathrm{mg} / \mathrm{kg}$ & 1 & Doi et $\mathrm{al}^{34}$ \\
\hline & Cohort B: 10 mg/kg & & \\
\hline & Cohort C: $30 \mathrm{mg} / \mathrm{kg}$ & & \\
\hline $\begin{array}{l}\text { FOLFOX4/paclitaxel-carboplatin/ } \\
\text { docetaxel + trebananib }\end{array}$ & Trebananib administered at $10 \mathrm{mg} / \mathrm{kg}$ & 1 & Mita et $\mathrm{al}^{40}$ \\
\hline \multirow[t]{2}{*}{ Paclitaxel/carboplatin + trebananib } & Paclitaxel $175 \mathrm{mg} / \mathrm{m}^{2} /$ carboplatin 6 AUC q3w + trebananib & $\mathrm{lb}$ & Casado et $\mathrm{al}^{41}$ \\
\hline & $15 \mathrm{mg} / \mathrm{kg}$ weekly and trebananib maintenance for 18 months & & \\
\hline \multirow[t]{2}{*}{ Topotecan + trebananib } & $4 \mathrm{mg} / \mathrm{m}^{2} \mathrm{qw}+10 \mathrm{mg} / \mathrm{kg} \mathrm{qw}$ & $\mathrm{lb}$ & NCT00770536 \\
\hline & $4 \mathrm{mg} / \mathrm{m}^{2} \mathrm{qw}+15 \mathrm{mg} / \mathrm{kg} \mathrm{qw}$ & & \\
\hline \multirow[t]{2}{*}{ Pegylated doxorubicin + trebananib } & $50 \mathrm{mg} / \mathrm{m}^{2} \mathrm{q} 4 \mathrm{w}+10 \mathrm{mg} / \mathrm{kg} \mathrm{qw}$ & & \\
\hline & $50 \mathrm{mg} / \mathrm{m}^{2} \mathrm{q} 4 \mathrm{w}+15 \mathrm{mg} / \mathrm{kg} \mathrm{qw}$ & & \\
\hline Paclitaxel + trebananib & $80 \mathrm{mg} / \mathrm{m}^{2}+10 \mathrm{mg} / \mathrm{kg} \mathrm{qw}$ & II & Karlan et al ${ }^{42}$ \\
\hline Paclitaxel + trebananib & $80 \mathrm{mg} / \mathrm{m}^{2}+3 \mathrm{mg} / \mathrm{kg} \mathrm{qw}$ & & \\
\hline Paclitaxel + placebo & $80 \mathrm{mg} / \mathrm{m}^{2} \mathrm{qw}$ & & \\
\hline
\end{tabular}

Abbreviations: 6 AUC, 6 area under the curve; q3w, every 3 weeks; qw, once a week; q4w, every 4 weeks. 
all participants. The other two ovarian cancer patients included in the study also received trebananib at the expansion phase cohort and experienced disease progression after 4 weeks. ${ }^{33}$

To expand our knowledge of trebananib safety and possible efficacy in a non-Caucasian population, a similar Phase I study was conducted in Japan. ${ }^{34}$ Eighteen patients were recruited in total - six in each of the three predesigned cohorts $(3 \mathrm{mg} / \mathrm{kg}$, $10 \mathrm{mg} / \mathrm{kg}$, and $30 \mathrm{mg} / \mathrm{kg}$ ). Trebananib administration and study design were similar to those of Herbst et al. ${ }^{33}$ Patients received a median of five and a half infusions of trebananib in the $3 \mathrm{mg} / \mathrm{kg}$ cohort and six infusions of the drug in the other two cohorts. No DLTs were observed during the study period and no patient discontinued treatment due to an adverse event.

The most common adverse events were peripheral edema (39\% of the patient population), fatigue, constipation, and pyrexia (each observed in $28 \%$ of patients). Hypertension was noted in four patients $(22 \%)$ but was Grade 1 or 2 . Increase of $\gamma$-glutamyltransferase was the most frequently reported Grade 3 or greater adverse event observed (four patients), but, according to the authors, this was not attributed to trebananib infusion. Serious adverse events were noted in three patients. Two of them received treatment in the $3 \mathrm{mg}$ / $\mathrm{kg}$ cohort and the other in the $30 \mathrm{mg} / \mathrm{kg}$ arm. These included ascites and pleural infusion in one patient, subclavian vein thrombosis and cholocystitis in the second, and anorexia in the third patient. Subclavian vein thrombosis was considered unrelated to trebananib treatment and was attributed to the central vein catheter of the patient.

The Japanese Phase I trial confirmed that trebananib has an acceptable safety profile when used as monotherapy in advanced solid tumors. Peripheral edema and fatigue are the primary adverse events encountered with trebananib use. Nausea was noted in a small percentage of patients ( $6 \%$ and $17 \%$, correspondingly) in the two studies, while the Japanese trial also indicated constipation and pyrexia as possible adverse events of trebananib.

In terms of efficacy, two patients - one in the $3 \mathrm{mg} / \mathrm{kg}$ cohort with a colon carcinoma and the other in the $30 \mathrm{mg} / \mathrm{kg}$ cohort with bladder cancer - achieved PR as the best response to treatment. The first patient with the colon cancer remained in trebananib treatment for $>2$ years. No ovarian carcinoma patients were included in this study.

\section{Phase I clinical trials of trebananib in combination with other antiangiogenic agents}

In the previously mentioned Phase I trials, trebananib showed acceptable safety for doses up to $30 \mathrm{mg} / \mathrm{kg}$ and evidence of efficacy as a monotherapy in solid tumors. Furthermore, its toxicity profile was different from that of anti-VEGF therapies. Taking into account the synergistic activity shown in preclinical models by dual inhibition of VEGF and Ang/Tie2 angiogenic pathways, ${ }^{25,35,36}$ further investigation of this combination in a clinical setting was warranted and is currently under examination. In one of these studies presented by Hong et $\mathrm{al}^{37}$ in the 2008 European Society for Medical Oncology Congress (NCT00861419), trebananib was combined with bevacizumab, sorafenib (only in renal cancer patients), or motesanib (AMG706, an oral multikinase inhibitor $)^{38}$ in advanced solid tumors. In the interim analysis presented, the combination of trebananib and bevacizumab was administered in 25 patients. Unfortunately, two of them suffering from head and neck carcinoma died due to fatal hemorrhagic event. No related thromboembolic events or hypertension Grade 3 or above was observed up to the interim analysis. The toxicity profile of other combinations was better, with one case of Grade 3 diarrhea reported for the trebananib-sorafenib arm. This combination was investigated in a Phase II study in renal cancer patients but did not prove clinical benefit. ${ }^{39}$

\section{Phase I and II clinical trials of trebananib in combination with chemotherapy}

The promising data of trebananib safety and efficacy as monotherapy from Phase I trials have driven further investigation of this agent in combination with cytotoxic chemotherapy. In the first exploratory study, ${ }^{40} 26$ patients enrolled to receive one of the three chemotherapy regimens (FOLFOX4, paclitaxel/carboplatin, or docetaxel) based on cancer type, along with weekly administration of trebananib. Trebananib dosage was determined at $10 \mathrm{mg} / \mathrm{kg}$, following a conservative strategy regarding the results from the Phase I monotherapy trials, since this dose maintains $90 \%$ effective drug concentration in the serum according to preclinical models. Only one patient with gynecological malignancy was included in the study in the arm of paclitaxel/carboplatin, diagnosed with concurrent breast and ovarian cancer. All patients received trebananib after the completion of the first cycle of chemotherapy.

Regarding safety, patients received a median of 14 cycles of trebananib and no DLTs were observed. All adverse events related to trebananib treatment were Grade 2 or lower. Most common were diarrhea and hypomagnesemia and only one patient developed peripheral edema. Regarding adverse events frequently encountered with anti-VEGF agents, in the docetaxel cohort two patients developed 
Grade 3 thromboembolic adverse events (pulmonary embolism and jugular vein thrombosis) that were considered possibly related to trebananib. Also, a patient with previously diagnosed hypertension under medication developed Grade 2 hypertension in the docetaxel cohort. Two deaths, one due to hepatic failure in the paclitaxel/carboplatin cohort and one due to respiratory failure in the docetaxel cohort, were not considered related to trebananib. It should be noted that no antitrebananib neutralizing antibodies were detected and combination treatment did not alter trebananib pharmacokinetics. By the evaluation of tumor response, one patient had a complete response, three patients had PR, and 13 patients had SD as best clinical response.

Based on the previously mentioned promising safety and efficacy results of trebananib in solid tumors, the drug was further evaluated in ovarian carcinoma patients in different clinical settings - more specifically, a Phase I trial testing trebananib with paclitaxel/carboplatin as firstline therapy in high-risk patients with ovarian cancer, ${ }^{41}$ a Phase II study administering trebananib and paclitaxel weekly in recurrent ovarian cancer patients, ${ }^{42}$ and a Phase Ib study combining pegylated doxorubicin or topotecan with trebananib (NCT00770536).

The Phase II study investigated the efficacy and safety of trebananib along with weekly paclitaxel in patients with recurrent ovarian cancer. One hundred and sixty-one patients were included in this study to receive weekly paclitaxel at $80 \mathrm{mg} / \mathrm{m}^{2}$ and were randomly assigned in a 1:1:1 fashion to also receive trebananib at $10 \mathrm{mg} / \mathrm{kg}(\operatorname{arm~A}), 3 \mathrm{mg} / \mathrm{kg}$ (arm B), or placebo (arm C). Patients were stratified according to prior use of antiangiogenic agents and time to progression from previous chemotherapy. At the time of analysis, no statistical significant difference was noted for PFS among three arms. The same applied for overall survival (OS) according to updated data.

Treatment discontinuation due to adverse events was more frequent in the investigational arms $(11 \%$ in $\operatorname{arm~} \mathrm{A}$ and $17 \%$ in arm B) in comparison with placebo (7\%). More frequently observed adverse events were peripheral edema, fatigue, nausea, diarrhea, and alopecia. Peripheral edema was more common in the investigational arms, but there was no trend for dose-related toxicity. Grade 3 and above adverse events that occurred more frequently in the investigational arms were hypokalemia, peripheral neuropathy, dyspnea, anorexia, and neutropenia. No death in arms A and B was related to trebananib. Common adverse events with antiVEGF therapies (hypertension, proteinuria) of Grade 3 and above were not observed, while thromboembolic events were similar among arms.
This study did not show a statistical improvement in PFS among arms, but a dose response effect was noted. Median PFS in patients receiving trebananib at $10 \mathrm{mg} / \mathrm{kg}$ increased 2.6 months relative to placebo, while the increase was 1.1 months for those receiving the drug at $3 \mathrm{mg} / \mathrm{kg}$. The dosedependent effect was confirmed by an exposure response analysis including patients from previous Phase I and II trials. ${ }^{43}$ The analysis suggested that the maximum clinical benefit was not reached by the administration of trebananib at $10 \mathrm{mg} / \mathrm{kg}$ and proposed the dosage of $15 \mathrm{mg} / \mathrm{kg}$ for further investigation. ${ }^{43,44}$ According to simulations of the used model, no more Grade 3 and above adverse events were anticipated with increased trebananib dose. ${ }^{43}$ However, this analysis was underpowered to detect a statistically significant difference in specific adverse events.

Despite concerns for safety in the $15 \mathrm{mg} / \mathrm{kg}$ trebananib dose, a Phase Ib study of weekly trebananib administration at this dosage in combination with paclitaxel $\left(175 \mathrm{mg} / \mathrm{m}^{2}\right)$ and carboplatin (6 area under the curve) every 3 weeks for six cycles, followed by trebananib maintenance up to 18 months, showed no DLTs for the 27 patients included in the interim analysis. ${ }^{41}$ All patients received trebananib and paclitaxel/ carboplatin combination as firstline treatment. Most common nonhematological adverse events were peripheral edema, fatigue nausea, and diarrhea, but none of them was Grade 3 or greater. Coadministration of chemotherapy did not alter trebananib pharmacokinetics and no antitrebananib neutralizing antibodies were detected.

Finally, a Phase Ib study investigated the feasibility of trebananib administration in patients with recurrent ovarian carcinoma in combination with either pegylated doxorubicin or topotecan (NCT00770536). The study has completed accrual of 103 patients and the results are anticipated. It is of interest that this is a dose escalation/de-escalation study including two parts. In each of the two study cohorts (pegylated doxorubicin and topotecan), the patients will also receive trebananib at either $10 \mathrm{mg} / \mathrm{kg}$ or $15 \mathrm{mg} / \mathrm{kg}$ to determine maximum tolerated dose. In the second part, patients in each of the two cohorts will receive trebananib at the maximum tolerated dose. Useful conclusions regarding the existence of a dose response effect of trebananib with these agents, as seen with paclitaxel, ${ }^{43}$ along with the safety of the combinations, will be drawn from this study.

\section{Phase III clinical trials of trebananib in ovarian cancer}

Based on the encouraging results of trebananib Phase I and II studies in ovarian carcinomas, the Trebananib in 
Table 2 Phase III studies of trebananib in ovarian carcinoma patients

\begin{tabular}{|c|c|c|c|c|c|}
\hline Study name & Regimen & PFS & $\mathbf{R R}$ & OS & Reference \\
\hline \multirow[t]{2}{*}{ TRINOVA-I } & Paclitaxel weekly + trebananib (15 mg/kg) & 7.2 & 38.4 & 19 & Monk et $\mathrm{a}^{47}$ \\
\hline & Paclitaxel weekly + placebo & 5.4 & 29.8 & 17.3 & \\
\hline \multirow[t]{2}{*}{ TRINOVA-2 } & Pegylated doxorubicin + trebananib $(15 \mathrm{mg} / \mathrm{kg})$ & NA & NA & NA & NCT0I $28 \mid 254$ \\
\hline & Pegylated doxorubicin + placebo & & & & \\
\hline \multirow[t]{2}{*}{ TRINOVA-3 } & Paclitaxel/carboplatin + trebananib $(15 \mathrm{mg} / \mathrm{kg})$ & NA & NA & NA & NCT0I493506 \\
\hline & Paclitaxel/carboplatin + placebo & & & & \\
\hline
\end{tabular}

Abbreviations: TRINOVA, Trebananib in Ovarian Cancer; PFS, progression-free survival; RR, response rate; OS, overall survival; NA, not applicable.

Ovarian Cancer (TRINOVA) program of Phase III studies was developed in order to provide clear evidence for the efficacy and safety of this drug in combination with standard chemotherapy either as a frontline treatment or as a treatment for recurrent disease (trebananib Phase III studies are summarized in Table 2).

TRINOVA-1 is a randomized, double-blind, Phase III trial evaluating weekly administration of paclitaxel plus trebananib/placebo in women with recurrent ovarian cancer. ${ }^{45}$ In this study, trebananib was administered as an intravenous infusion at a dosage of $15 \mathrm{mg} / \mathrm{kg}$, while paclitaxel was given weekly at $80 \mathrm{mg} / \mathrm{m}^{2}$ in a 3 weeks on $/ 1$ week off schema. The primary end point of the study was PFS, while OS, objective response rate, pharmacokinetics, and incidence of adverse events were secondary outcome measures. Nine hundred and nineteen women were recruited until November 2012. Eligible patients should have received at least one prior platinum-based regimen and up to three lines of chemotherapy in total. Patients with platinum refractory disease (recurrence during or within 6 months of the beginning of platinumbased firstline chemotherapy) and those with a platinum-free interval (PFI) $>12$ months from their last platinum-based therapy were excluded from the study. At cutoff date, data for the primary PFS analysis and interim OS analysis were included. Study results were presented by Monk et $\mathrm{al}^{45,46}$ in the 2013 European Cancer Organisation conference demonstrating clear clinical benefit for the addition of trebananib in weekly paclitaxel chemotherapy in this clinical setting. More specifically, there was a statistically significant increase in PFS from a median of 5.4 months in the control group to 7.2 months in the experimental group (hazard ratio $[\mathrm{HR}]=0.66$, $P<0.001)$ and an analogous improvement in overall response rate favoring the patients who received trebananib (38.4\% vs $29.8 \%, P<0.0071)$. It should be noted that the PFS improvement was consistent both in patients with platinumresistant disease (PFI $<6$ months) and in those with partial platinum-sensitive recurrence (PFI 6-12 months). Despite a trend of improved OS in the trebananib arm being shown (median 19.0 months vs 17.3 months in the control arm), the difference did not reach statistical significance $(\mathrm{HR}=0.86$, $P=0.19)$ at the interim analysis, and mature survival data are anticipated during 2014.

Regarding safety, no major difference in Grade 3 or greater adverse events was noted between the two study groups. Adverse events that were increased in the trebananib arm included localized edema, pleural effusion, ascites, and generalized edema, as shown in previous studies, as well as peripheral neuropathy, attributed by the authors to the increased paclitaxel exposure of the patients in the experimental arm. Again, no major difference in adverse events related to anti-VEGF therapies was noted with trebananib use.

\section{Conclusion}

The results of TRINOVA-1 suggest that targeting the Ang/ Tie2 pathway is associated with a clinical benefit in ovarian carcinoma patients. Nevertheless, several clinical questions regarding the optimal use of trebananib need further clarification.

The best clinical setting for the use of trebananib remains to be answered. Could this agent also be beneficial along with frontline chemotherapy? TRINOVA-3, a randomized Phase III trial of paclitaxel/carboplatin plus trebananib at $15 \mathrm{mg} / \mathrm{kg}$ or placebo followed by trebananib/placebo maintenance for 18 months in women with International Federation of Gynecology and Obstetrics Stage III-IV ovarian cancer, will delineate this issue. The study has completed recruitment and results are anticipated eagerly.

The second issue raised by TRINOVA-1 results is the exact place of trebananib in ovarian cancer treatment, since the other antiangiogenic agent bevacizumab has proven clinical efficacy both in the frontline setting and in disease recurrence. This is definitely a more complex matter and requires more clinical trials with both agents and a deeper knowledge of bevacizumab and trebananib resistance mechanisms in order to be answered. Since the Ang/Tie2 pathway could be implicated in the development of bevacizumab resistance mechanisms ${ }^{47}$ a rational approach would be to use trebananib in disease recurrence after bevacizumab failure. 
Furthermore, trebananib toxicity profile with high incidence of peripheral edema and ascites may not be suitable for patients with advanced disease, deteriorated clinical status, or preexisting ascites. However, Monk et $\mathrm{al}^{48}$ have recently put forward a provocative idea regarding the optimal use of bevacizumab in ovarian cancer. They proposed that the ratio of median bevacizumab administration to the median PFS increase in months in the conducted trials, indicating how many months bevacizumab should be administered to gain a 1 month increase in PFS, could be a measure for its use. This ratio no doubt favors the use of bevacizumab in disease recurrence. Finally, TRINOVA-1 allowed prior exposure to antiangiogenic agents for the enrolled patients. However, the great degree of variability in the PFS data for these patients renders the results inconclusive at this primary analysis. More mature data of the study and new studies are definitely required to shed light on the optimal sequencing of antiangiogenic therapies in ovarian cancer.

In conclusion, trebananib is a new targeted therapy active in recurrent ovarian cancer patients in combination with paclitaxel. It definitely enhances our therapeutic armamentarium for this difficult-to-treat disease and provides a new potential for patients with platinum-resistant recurrence. The availability of two active antiangiogenic agents in the same clinical setting (bevacizumab and trebananib), though, creates questions regarding their optimal sequencing and/or combination. Hopefully, data from current and future studies will provide appropriate answers to guide the clinicians and benefit the patients.

\section{Disclosure}

Aristotle Bamias has received research support from Amgen. All other authors declare no conflicts of interest in this work.

\section{References}

1. Alvarez AA, Krigman HR, Whitaker RS, Dodge RK, Rodriguez GC. The prognostic significance of angiogenesis in epithelial ovarian carcinoma. Clin Cancer Res. 1999;5(3):587-591.

2. Bamias A, Pignata S, Pujade-Lauraine E. Angiogenesis: a promising therapeutic target for ovarian cancer. Crit Rev Oncol Hematol. 2012;84(3):314-326.

3. Tsiatas ML, Gyftaki R, Liacos C, et al. Study of T lymphocytes infiltrating peritoneal metastases in advanced ovarian cancer: associations with vascular endothelial growth factor levels and prognosis in patients receiving platinum-based chemotherapy. Int J Gynecol Cancer. 2009;19(8):1329-1334.

4. Fujimoto J, Sakaguchi H, Aoki I, Khatun S, Tamaya T. Clinical implications of expression of vascular endothelial growth factor in metastatic lesions of ovarian cancers. Br J Cancer. 2001;85(3):313-316.

5. Burger RA, Sill MW, Monk BJ, Greer BE, Sorosky JI. Phase II trial of bevacizumab in persistent or recurrent epithelial ovarian cancer or primary peritoneal cancer: a gynecologic oncology group study. J Clin Oncol. 2007;25(33):5165-5171.
6. Cannistra SA, Matulonis UA, Penson RT, et al. Phase II study of bevacizumab in patients with platinum-resistant ovarian cancer or peritoneal serous cancer. J Clin Oncol. 2007;25(33):5180-5186.

7. Burger RA, Brady MF, Bookman MA, et al. Incorporation of bevacizumab in the primary treatment of ovarian cancer. $N$ Engl J Med. 2011;365(26):2473-2483.

8. Perren TJ, Swart AM, Pfisterer J, et al. A Phase 3 trial of bevacizumab in ovarian cancer. N Engl J Med. 2011;365(26):2484-2496.

9. Aghajanian C, Blank SV, Goff BA, et al. OCEANS: a randomized, double-blind, placebo-controlled phase III trial of chemotherapy with or without bevacizumab in patients with platinum-sensitive recurrent epithelial ovarian, primary peritoneal, or fallopian tube cancer. J Clin Oncol. 2012;30(17):2039-2045.

10. Pujade-Lauraine E, Hilpert F, Weber B, et al. AURELIA: a randomized phase III trial evaluating bevacizumab (BEV) plus chemotherapy (CT) for platinum (PT)-resistant recurrent ovarian cancer (OC). J Clin Oncol. 2012;30(Suppl):LBA5002.

11. Pujade-Lauraine E, Hilpert F, Weber B, et al. Bevacizumab combined with chemotherapy for platinum-resistant recurrent ovarian cancer: the AURELIA open-label randomized phase III trial. J Clin Oncol. Epub March 17, 2014.

12. Burger RA, Brady MF, Bookman MA, et al. Risk factors for GI adverse events in a phase III randomized trial of bevacizumab in first-line therapy of advanced ovarian cancer: a Gynecologic Oncology Group study. J Clin Oncol. Epub March 17, 2014.

13. Randall LM, Monk BJ. Bevacizumab toxicities and their management in ovarian cancer. Gynecol Oncol. 2010;117(3):497-504.

14. Hanahan D, Weinberg RA. The hallmarks of cancer. Cell. 2000;100(1):57-70.

15. Hanahan D, Weinberg RA. Hallmarks of cancer: the next generation. Cell. 2011;144(5):646-674.

16. Carmeliet P, Jain RK. Angiogenesis in cancer and other diseases. Nature. 2000;407(6801):249-257.

17. Hillen F, Griffioen AW. Tumour vascularization: sprouting angiogenesis and beyond. Cancer Metastasis Rev. 2007;26(3-4):489-502.

18. Cascone T, Heymach JV. Targeting the angiopoietin/Tie2 pathway: cutting tumor vessels with a double-edged sword? J Clin Oncol. 2012;30(4):441-444.

19. Kunkel P, Ulbricht U, Bohlen P, et al. Inhibition of glioma angiogenesis and growth in vivo by systemic treatment with a monoclonal antibody against vascular endothelial growth factor receptor-2. Cancer Res. 2001;61(18):6624-6628.

20. Oliner J, Min H, Leal J, et al. Suppression of angiogenesis and tumor growth by selective inhibition of angiopoietin-2. Cancer Cell. 2004;6(5):507-516.

21. Shimamoto G, Gegg C, Boone T, Queva C. Peptibodies: a flexible alternative format to antibodies. MAbs. 2012;4(5):586-591.

22. Maisonpierre PC, Suri C, Jones PF, et al. Angiopoietin-2, a natural antagonist for Tie2 that disrupts in vivo angiogenesis. Science. 1997;277(5322):55-60.

23. Etoh T, Inoue H, Tanaka S, Barnard GF, Kitano S, Mori M. Angiopoietin-2 is related to tumor angiogenesis in gastric carcinoma: possible in vivo regulation via induction of proteases. Cancer Res. 2001;61(5):2145-2153.

24. Ahmad SA, Liu W, Jung YD, et al. The effects of angiopoietin-1 and -2 on tumor growth and angiogenesis in human colon cancer. Cancer Res. 2001;61(4):1255-1259.

25. Coxon A, Bready J, Min H, et al. Context-dependent role of angiopoietin-1 inhibition in the suppression of angiogenesis and tumor growth: implications for AMG 386, an angiopoietin-1/2-neutralizing peptibody. Mol Cancer Ther. 2010;9(10):2641-2651.

26. Falcón BL, Hashizume H, Koumoutsakos P, et al. Contrasting actions of selective inhibitors of angiopoietin-1 and angiopoietin-2 on the normalization of tumor blood vessels. Am J Pathol. 2009;175(5): 2159-2170.

27. Holash J, Wiegand SJ, Yancopoulos GD. New model of tumor angiogenesis: dynamic balance between vessel regression and growth mediated by angiopoietins and VEGF. Oncogene. 1999;18(38):5356-5362. 
28. Thurston G, Suri C, Smith K, et al. Leakage-resistant blood vessels in mice transgenically overexpressing angiopoietin-1. Science. 1999;286(5449):2511-2514.

29. Bicknell R, Harris AL. Anticancer strategies involving the vasculature: vascular targeting and the inhibition of angiogenesis. Semin Cancer Biol. 1992;3(6):399-407.

30. Heath VL, Bicknell R. Anticancer strategies involving the vasculature. Nat Rev Clin Oncol. 2009;6(7):395-404.

31. Hata K, Udagawa J, Fujiwaki R, Nakayama K, Otani H, Miyazaki K. Expression of angiopoietin-1, angiopoietin-2, and Tie2 genes in normal ovary with corpus luteum and in ovarian cancer. Oncology. 2002;62(4):340-348.

32. Rosen LS, Hong D, Chap L, et al. First-in-human study of AMG 386, a selective angiopoietin 1/2-neutralizing peptibody, in adult patients with advanced solid tumors. J Clin Oncol. 2007;25(18) (Supp1):3522.

33. Herbst RS, Hong D, Chap L, et al. Safety, pharmacokinetics, and antitumor activity of AMG 386, a selective angiopoietin inhibitor, in adult patients with advanced solid tumors. J Clin Oncol. 2009;27(21):3557-3565.

34. Doi T, Ohtsu A, Fuse N, et al. Phase 1 study of trebananib (AMG 386), an angiogenesis targeting angiopoietin-1/2 antagonist, in Japanese patients with advanced solid tumors. Cancer Chemother Pharmacol. 2013;71(1):227-235.

35. Hashizume H, Falcon BL, Kuroda T, et al. Complementary actions of inhibitors of angiopoietin-2 and VEGF on tumor angiogenesis and growth. Cancer Res. 2010;70(6):2213-2223.

36. Brown JL, Cao ZA, Pinzon-Ortiz M, et al. A human monoclonal antiANG2 antibody leads to broad antitumor activity in combination with VEGF inhibitors and chemotherapy agents in preclinical models. $\mathrm{Mol}$ Cancer Ther. 2010;9(1):145-156.

37. Hong D, Gordon M, Appleman L, et al. Interim results from a Phase $1 \mathrm{~b}$ study of safety, pharmacokinetics (PK) and tumor response of the angiopoietin 1/2-neutralizing peptibody AMG 386 in combination with AMG 706, bevacizumab (B) or sorafenib (S) in advanced solid tumors. Ann Oncol. 2008;19(Suppl 8):viii153-viii165.

38. Polverino A, Coxon A, Starnes C, et al. AMG 706, an oral, multikinase inhibitor that selectively targets vascular endothelial growth factor, platelet-derived growth factor, and kit receptors, potently inhibits angiogenesis and induces regression in tumor xenografts. Cancer Res. 2006;66(17):8715-8721.
39. Rini B, Szczylik C, Tannir NM, et al. AMG 386 in combination with sorafenib in patients with metastatic clear cell carcinoma of the kidney: a randomized, double-blind, placebo-controlled, phase 2 study. Cancer. 2012;118(24):6152-6161.

40. Mita AC, Takimoto CH, Mita M, et al. Phase 1 study of AMG 386, a selective angiopoietin 1/2-neutralizing peptibody, in combination with chemotherapy in adults with advanced solid tumors. Clin Cancer Res. 2010;16(11):3044-3056.

41. Casado A, Oaknin A, Baurain J, et al. Phase Ib study of AMG 386 in combination with paclitaxel (P) and carboplatin (C) in high-risk stage I and stages II-IV epithelial ovarian, primary peritoneal, or fallopian tube cancers. J Clin Oncol. 2012;30(Suppl):5038.

42. Karlan BY, Oza AM, Richardson GE, et al. Randomized, double-blind, placebo-controlled phase II study of AMG 386 combined with weekly paclitaxel in patients with recurrent ovarian cancer. J Clin Oncol. 2012;30(4):362-371.

43. Lu JF, Rasmussen E, Karlan BY, et al. Exposure-response relationship of AMG 386 in combination with weekly paclitaxel in recurrent ovarian cancer and its implication for dose selection. Cancer Chemother Pharmacol. 2012;69(5):1135-1144.

44. Sood AK, Coleman RL, Ellis LM. Moving beyond anti-vascular endothelial growth factor therapy in ovarian cancer. J Clin Oncol. 2012;30(4):345-347.

45. Monk B, Poveda A, Vergote I, et al. A phase III, randomized, double-blind trial of weekly paclitaxel plus the angiopoietin 1 and 2 inhibitor, trebananib, or placebo in women with recurrent ovarian cancer: TRINOVA-1. Presented at: European Cancer Congress 2013 (ECCO-ESMO-ESTRO); September 27-October 1, 2013; Amsterdam, the Netherlands. Abstract LBA41.

46. Monk BJ, Poveda A, Vergote I, et al. Anti-angiopoietin therapy with trebananib for recurrent ovarian cancer (TRINOVA-1): a randomised, multicentre, double-blind, placebo-controlled phase 3 trial. Lancet Oncol. 2014;15(8):799-808.

47. Moreno Garcia V, Basu B, Molife LR, Kaye SB. Combining antiangiogenics to overcome resistance: rationale and clinical experience. Clin Cancer Res. 2012;18(14):3750-3761.

48. Monk BJ, Pujade-Lauraine E, Burger RA. Integrating bevacizumab into the management of epithelial ovarian cancer: the controversy of front-line versus recurrent disease. Ann Oncol. 2013;24 Suppl 10:x53-x58.
OncoTargets and Therapy

\section{Publish your work in this journal}

OncoTargets and Therapy is an international, peer-reviewed, open access journal focusing on the pathological basis of all cancers, potential targets for therapy and treatment protocols employed to improve the management of cancer patients. The journal also focuses on the impact of management programs and new therapeutic agents and protocols on

\section{Dovepress}

patient perspectives such as quality of life, adherence and satisfaction The manuscript management system is completely online and includes a very quick and fair peer-review system, which is all easy to use. Visit http://www.dovepress.com/testimonials.php to read real quotes from published authors. 